\title{
NUCLEAR LOGALIZATION OF PARATHYMOSIN IN RAT AND HUMAN HEPATOCYTES. A LIGHT AND ULTRASTRUCTURAL STUDY
}

\author{
Tomas Garcia-Caballero ${ }^{1}$, Elena ROSON, Rosalia Gallego, Maximo \\ FRAGA, Fernando DOMINGUEZ and Andres BEIRAS \\ Departamentos de Ciencias Morfologicas (TG-C, ER, RG, AB), Anatomia Patologica (MF) y \\ Fisiologia (FD), Facultad de Medicina, Universidad de Santiago, S. Francisco \\ s/n. 15705 Santiago de Compostela, Spain
}

Received for publication September 30, 1992 and in revised form March 2, 1993

\begin{abstract}
Parathymosin (ParaT) is an almost ubiquitious peptide first isolated from rat thymus, the highest concentration being in the liver. Its subcellular localization is controversial and both a cytoplasmic and a nuclear distribution have been reported. The aim of the present work was to study the intracellular localization of ParaT in rat and human livers by light immunohistochemistry and immunoelectron microscopy procedures. Both in rat and human livers immunostaining for $P$ ara $T$ was found in the nuclei of hepatocytes, the nucleoli and the cytoplasms being negative. The intensity of immunostaining was variable from cell to cell, but virtually all hepatocytes were positive. No immunoreactivity was found in non-parenchymal cells. Immunogold labelling confirmed these results and showed that ParaT immunoreactivity was associated with both euchromatin and heterochromatin. Our results demonstrate that ParaT is localized in the nucleus of hepatocytes and support the idea that the inactivation of the cytoplasmic enzyme phosphofructokinase-1 is not the unique function of ParaT.
\end{abstract}

Parathymosin (ParaT) is a peptide of 101-105 amino acids first isolated from rat thymus (11). It was named parathymosin because it is similar to prothymosin alpha in size and amino acid composition (11), although they present only limited sequence homology, primarily in the C-terminal region $(8,13)$. Both peptides were initially claimed for an immunomodulatory role acting as thymic hormones, but this role has been questioned because of their ubiquitous distribution $(4,10,11,20)$ and their lack of a hydrophobic signal sequence necessary for their secretion $(5,7,9)$. ParaT concentration in the different tissues studied is approximately reciprocal to that of prothymosin alpha, the former reaching the highest concentration in liver, whereas the content of prothymosin alpha in this organ is very low $(1,4,10)$. At present, the identity of ParaT with a $11.5 \mathrm{kD} \mathrm{Zn}^{2+}$-binding protein, originally found in rat liver by its ability to inactivate phosphofructokinase-1 (3), was establish-

This work was supported by a grant from Xunta de Galicia (XUGA 20808B90).

1 To whom correspondence and reprint request should be sent. ed (19). However, the main physiological function of ParaT has not been found (2). Its intracellular localization is also controversial and both a nuclear (2, $6,22)$ and a cytoplasmic distribution $(3,16,21)$ have been proposed. The aim of the present work was to determine, by light immunohistochemical and immunogold labelling procedures, the subcellular distribution of ParaT in rat and human hepatocytes. A clear knowledge of this distribution is essential to clarify the precise role of ParaT.

\section{MATERIALS AND METHODS}

\section{ParaT antiserum}

A polyclonal antibody to ParaT (generously given by Dr. B. L. Horecker) was employed. On the basis of competition experiments with peptide fragments derived from ParaT, the major epitope of this antibody appears to comprise a region in ParaT including amino acids residues 26-32 (14). As the ParaT sequence homologue with synthetic thymosin alpha-1 is comprised between residues $14-25$ (8), the antibody showed crossreactivity with neither thymosin 
alpha-1 nor prothymosin alpha.

Light immunohistochemical staining

Normal human liver $(n=10)$ was obtained from biopsies or recent autopsies. Adult male and female Sprague-Dawley rats $(\mathrm{n}=10)$ weighing $200-250 \mathrm{~g}$ were maintained on a $12 \mathrm{hr}$ light-dark cycle and provided with water and food ad libitum. They were rapidly decapitated after being stunned. Samples were fixed by immersion in $4 \%$ buffered formaldehyde for $24 \mathrm{hr}$, dehydrated, and embedded in paraffin routinely. Sections $4 \mu \mathrm{m}$ thick were mounted on gelatin chromealum coated slides and immunostained by the avidin-biotinperoxidase complex (ABC) procedure of $\mathrm{Hsu}$ et al. (12). Briefly, after blocking steps (hydrogen peroxide $0.3 \%$ for $10 \mathrm{~min}$ and normal goat serum 1:10 for $30 \mathrm{~min}$ ) the sections were consecutively incubated at room temperature in : 1) rabbit antiserum to ParaT at a dilution of $1: 2000$ (for $1 \mathrm{hr}$ ); 2) biotinylated antibody to rabbit immunoglobulins raised in goat (Dakopatts, Glostrup, Denmark) at a dilution of $1: 400$ (for $30 \mathrm{~min}$ ); 3) avidin-biotin-peroxidase complex (Vectastain Elite Kit, Vector, Burlingame, Ca) prepared according to the protocol provided by the manufacturer (for $30 \mathrm{~min}$ ); 4) treatment for $5 \mathrm{~min}$ with $0.06 \%(w / v) \quad 3,3^{\prime}$-diaminobenzidine tetrahydrochloride (DAB) (Sigma, St Louis, MO) and 0.003\% $(\mathrm{v} / \mathrm{v})$ hydrogen peroxide. Between steps, the sections were rinsed with PBS ( $\mathrm{pH} 7.4$ ) twice for 5 min After step 4 the sections were rinsed with distilled water, dehydrated, cleared, and mounted. All dilutions were done in PBS ( $\mathrm{pH}$ 7.4). Controls were performed by either: a) substitution of the immune antiserum with normal (non-immune) rabbit serum or b) using PBS in place of one of the other incubation steps. In both cases, negligible immunoreactivity was found.

\section{Ultrastructural immunocytochemistry}

For immunoelectron microscopic techniques, normal rat liver samples were fixed in $2 \%$ paraformaldehyde- $0.2 \%$ glutaraldehyde in $0.1 \mathrm{M}$ sodium phosphate buffer ( $\mathrm{pH}$ 7.4). After washing in phosphate buffer, the specimens were dehydrated in graded ethanol series and embedded in LR White (The London Resin Co, Basingstoke, UK). Polymerization was done at $60^{\circ} \mathrm{C}$ for $8 \mathrm{hr}$, avoiding contact bet- ween resin and oxygen. Ultrathin sections $(60 \mathrm{~nm}$ thick) were collected on uncoated 300-mesh nickel grids. Labelling procedures were performed at room temperature on floating grids. The next protocol was followed: 1) normal goat serum $1: 50$ in $0.1 \mathrm{M}$ PBS containing $0.5 \%$ ovalbumin (5 min), 2) ParaT antiserum (overnight at $1: 100-1: 500$ in $0.1 \mathrm{M}$ PBS containing $1 \%$ ovalbumin), 3) $0.1 \mathrm{M}$ PBS $(5 \times 1 \mathrm{~min})$, 4) $0.2 \%$ ovalbumin in $0.05 \mathrm{M}$ Tris- $\mathrm{HCl}, \mathrm{pH} 7.6$ (5 min.), 5) goat-anti-rabbit lgG-20 nm gold conjugates (BioCell, Cardiff, UK) diluted $1: 20$ in $0.02 \mathrm{M}$ Tris- $\mathrm{HCl}, \mathrm{pH} 7.6$, containing $1 \%$ ovalbumin $(1 \mathrm{hr})$, 6) $0.02 \mathrm{M}$ Tris- $\mathrm{HCl} \mathrm{pH} 7.6(5 \times 1 \mathrm{~min})$, 7)distilled water $(5 \times 1 \mathrm{~min}), 8) 2 \%$ uranyl acetate $(2 \mathrm{~min}), 9)$ distilled water $(5 \mathrm{~min})$. Controls performed in the same way as for immunohistochemistry at light microscopic level gave a negligible reaction.

\section{RESULTS}

Both in rat and human livers, ParaT immunoreactivity was found in the nuclei of hepatocytes, the cytoplasms being negative (Figs. 1, 2). The immunostaining was homogeneously distributed throughout the hepatic lobule (Figs. 1, 2). At great magnification, it can be shown that virtually all hepatocytes were positive, the intensity of nuclear immunostaining being variable and the nucleoli constantly negative (Fig. 3). No immunorectivity was seen in the sinusoidal lining cells (Fig. 3).

The immunogold technique confirmed the nuclear localization of ParaT in the hepatocytes (Fig. 4). The immunolabelling was found both in euchromatin and heterochromatin, although a tendency to exclude the peripheral and perinucleolar heterochromatin regions was observed. The nucleoli presented only occasional gold granules, but always associated to nucleoplasm interstices. The scarce immunolabelling found in the cytoplasm (Fig. 4) was not greater than the background seen in the control (not shown).

Fig. 1. In rat liver ParaT immunoreactivity is localized in the nuclei of hepatocytes, being diffusely distributed throughout the hepatic lobule (CV: central vein, arrows: portal spaces). $\times 50 \quad \mathrm{Bar}=200 \mu \mathrm{m}$.

Fig. 2. A similar pattern is found in human liver, where ParaT immunostaining is also homogeneously distributed and restricted to the nuclei of hepatocytes (CV: central vein, arrows: portal spaces). $\times 140 \mathrm{Bar}=100 \mu \mathrm{m}$.

Fic. 3. At great magnification it can be seen that virtually all hepatocytes are positive, showing varying intensity of staining. Nucleoli were excluded. $\times 400$ Bar $=30 \mu \mathrm{m}$.

Fic. 4. Immunolabelling for ParaT is found is the hepatocyte nucleus, both in euchromatin and heterochromatin. The gold granules observed in nucleolus are associated to interstices of nucleoplasm. $\times 18,500 \quad$ Bar $=1 \mu \mathrm{m}$. 


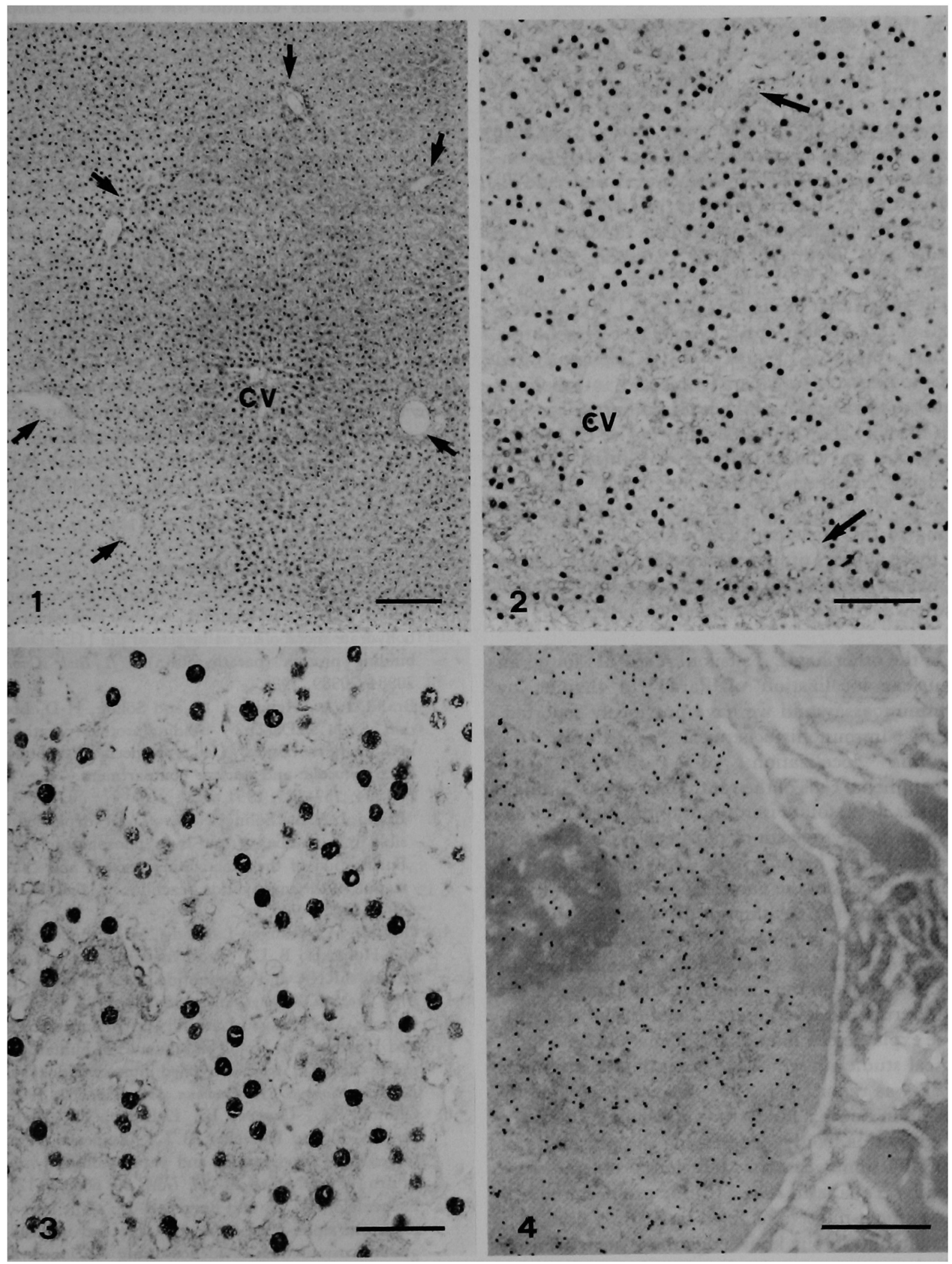




\section{DISCUSSION}

In this report we have demonstrated by immunochemical techniques, both at light and ultrastructural level, the nuclear localization of ParaT in hepatocytes. The antibody used reacted with both human and rat ParaT. This was expected because protein sequencing data have shown that ParaT is conserved in evolution $(5,15)$. The human molecule differs from the rat molecule by less than $10 \%$ (17), the region of the major epitope of the antibody (see materials and methods) being common for both species.

The subcellular localization of ParaT is controversial and both a nuclear and a cytoplasmic distribution have been reported. Watts et al. (22) demonstrated that radioiodinated rat ParaT injected into oocytes migrates to the nucleus and accumulates to a concentration of more than ten times that in the cytoplasm. Also relevant was the experiment of Clinton et al. (6) who, using constructs coding for ParaT linked to human growth hormone, also demonstrated ParaT targeting to the nucleus of HeLa S3 cells. Furthermore, these authors proposed that a possible candidate for a nuclear localization signal in the human ParaT molecule was the region comprised from residue 91 to 94 (6).

On the other hand, Tsitsiloni et al. (21) found an extranuclear localization of ParaT in thymus by radioimmunoassay and we have previously reported, by light immunohistochemical procedures, the cytoplasmic localization of ParaT in the reticuloepithelial cells of the rat thymus (16). Similarly, Brand et al. (2) found immunostaining for ParaT exclusively in the cytoplasm of most cell types that they studied, including hepatocytes. Only intestinal crypt cells and duct cells of the parotid gland showed nuclear immunostaining (2). Although these results could be explained by a cell type-specific distribution of ParaT, the disagreement in the ParaT localization in hepatocytes between our present results (both at light and ultrastructural level) and those of Brand et al. (2), could be explained by fixation problems. In both morphological studies in which a cytoplasmic distribution of ParaT was reported, $(2,16)$, fixation was done by immersion in Bouin's fluid. It is known that lipids are dissolved by this fixative, increasing the possibility of antigen diffusion. Similar diffusion problems were reported by Takahashi et al. (18) for proliferating cell nuclear antigen in sections fixed in Bouin's fluid.

To the best of our knowledge there have been no published reports concerning immunoelectron localization of ParaT. By indirect immunofluorescence, Clin- ton et al. (6) found that the nuclear staining of ParaT in HeLa S3 cells excluded the nucleolar compartment. This finding is in agreement with our results in hepatocytes, both at light and electron microscopy.

In addition to the known ParaT ability to inactivate phosphofructokinase-1 (3), it has been reported that aldolase and several other enzymes involved in carbohydrate metabolism also bind to ParaT in a $\mathrm{Zn}^{2+}$. dependent manner (1). Our finding of a nuclear localization of ParaT seems to be in disagreement with these activities related to cytoplasmic enzymes. However, the lack of significant amounts of ParaT in skeletal muscle and erythrocytes, both with high contents of phosphofructokinase-1, makes it unlikely that the inactivation of phosphofructokinase- 1 reflects the main physiological function of ParaT (2). Assuming the nuclear localization of ParaT, alternative functions have been proposed: a chaperone function (perhaps for cations) or a possible role in transcriptional activation (22). Further studies will be necessary to clarify the precise role of ParaT in nuclear physiology.

\section{REFERENCES}

1. Brand, I. A. and Heinickel, A.: Key enzymes of carbohydrate metabolism as targets of the $11.5-\mathrm{kDa} \mathrm{Zn} 2+$ binding protein (parathymosin). J. Biol. Chem. 31; 20984-20989, 1991.

2. Brand, I. A., Heinickel, A. and Söling, H-D. Localization of a $11.5 \mathrm{kDa} \mathrm{Zn2}+$-binding protein (parathymosin) in different rat tissues. Cell type-specific distribution between cytosolic and nuclear compartment. Eur. J. Cell. Biol. 54; 157-165, 1991.

3. Brand, I. A and Söling, H-D.: Zn2+-dependent reversible inactivation of rat liver phosphofructokinase-1. Purification of the inactivating protein and characterization of the inactivation reaction. J. Biol. Chem. 261; 5892-5900, 1986.

4. Clinton, M., Frangou-Lazaridis, M., Panneerselvam, C. and Horecker, B. L.: Prothymosin alpha and parathymosin: mRNA and polypeptide levels in rodent tissues. Arch. Biochem. Biophys. 269; 256-263, 1989.

5. Clinton, M., Frangou-Lazaridis, M., Panneerselvam, C. and Horecker, B. L.: The sequence of human parathymosin deduced from a cloned human kidney cDNA. Biochem. Biophys. Res. Commun. 158; 855-862, 1989.

6. Clinton, M., Graeve, L., El-Dorry, H., RodriguezBoulan, E. and Horecker, B. L.: Evidence for nuclear targeting of prothymosin and parathymosin synthesized in situ. Proc. Natl. Acad. Sci. USA. 88; 6608-6612, 1991.

7. Eschenfeldt, W. H. and Berger, S. L.: The human prothymosin alpha gene is polymorphic and induced upon growth stimulation: evidence using a cloned cDNA. Proc. Natl. Acad. Sci. USA. 83; 9403-9407, 1986.

8. Frangou-Lazaridis, M., Clinton, M., Goodall, G. J. and 
Horecker, B. L.: Prothymosin alpha and parathymosin: amino acid sequences deduced from the cloned rat spleen cDNAs. Arch. Biochem. Biophys. 263; 305-310, 1988.

9. Goodall, G. J., Dominguez, F. and Horecker, B. L.: Molecular cloning of cDNA for human prothymosin alpha. Proc. Natl. Acad. Sci. USA. 83; 8926-8928, 1986.

10. Haritos, A. A., Tsolas, $O$. and Horecker, B. L.: Distribution of prothymosin alpha in rat tissues. Proc. Natl. Acad. Sci. USA. 81; 1391-1393, 1984.

11. Haritos, A. A., Salvin, S. B., Blacher, R., Stein, S. and Horecker, B. L.: Parathymosin alpha: a peptide from rat tissues with structural homology to prothymosin alpha. Proc. Natl. Acad. Sci. USA. 82; 1050-1053, 1985.

12. Hsu, S. M., Raine, L. and Fanger, H.: The use of avidin-biotin-peroxidase complex (ABC) in immunoperoxidase techniques: a comparison between $\mathrm{ABC}$ and unlabelled antibody (PAP) procedures. J. Histochem. Cytochem. 29; 577-580, 1981.

13. Komiyama, T., Pan, L. X., Haritos, A. A., Wideman, J. W., Pan, Y. E., Chang, M., Rogers, I. and Horecker, B. L.: The primary structure of rat parathymosin. Proc. Natl. Acad. Sci. USA. 83; 1242-1245, 1986.

14. Panneerselvam, C., Caldarella, J. and Horecker, B. L.: A radioimmunoassay for parathymosin. J. Immunol. Methods 104; 131-136, 1987.

15. Panneerselvam, C., Clinton, M., Wellner, D. and Horecker, B. L.: Bovine parathymosin: amino acid sequence and comparison with rat parathymosin. Biochem. Biophys. Res. Commun. 155; 539-545, 1988.

16. Roson, E., Garcia-Caballero, T., Heimer, E. P., Felix,
A. M. and Dominguez, F.: Cellular distribution of prothymosin alpha and parathymosin in rat thymus and spleen. J. Histochem. Cytochem. 38; 1889-1894, 1990.

17. Szabo, P., Clinton, M., Macera, M. and Horecker, B. L.: Localization of the gene coding for parathymosin to chromosome 17 in humans. Cytogenet. Cell. Genet. 50; 91-92, 1989.

18. Takahashi, H., Oishi, Y., Chuang, S-S. and Morii, S.: Effects of tissue fixation and processing on proliferating cell nuclear antigen (PCNA) immunohistochemistry. Acta Pathol. Jap. 42; 621-623.

19. Trompeter, H-I., Brand, I. A. and Söling, H-D: The primary sequence of the PFK-1 inactivating zinc-binding protein as deduced from cDNA sequencing. Identity of the zinc-binding protein with rat parathymosin. FEBS Lett. 253; 63-66, 1989.

20. Tsitsiloni, O. E., Yialouris, P. P., Heimer, E. P., Felix, A. M., Evangelatos, G. P., Soteriadis-Vlahos, C., Stiakakis, J., Hannapel, E. and Haritos, A. A.: A radioimmunoassay for parathymosin alpha using antibodies to synthetic N-terminal peptide 1-30. J. Immunol. Methods 113 ; 175-184, 1988.

21. Tsitsiloni, O. E., Yilouris, P.P., Echner, H., Voelter, W. and Haritos, A. A.: Evidence for the extranuclear localization of thymosins in thymus. Experientia 48; 398402, 1992.

22. Watts, J. D., Cary, P. D., Sautiere, P. and Crane-Robinson, C.: Thymosins: both nuclear and cytoplasmic proteins. Eur. J. Biochem. 192; 643-651, 1990. 